\title{
Synthesis of 2-Pyrazoline-5-Carboxylic Acid Derivatives Using Trimethylsilyldiazomethane
}

\author{
Jin Hee Ahn," Hye-Min Kim,' Seung Kyu Kang, Jae Du Ha, Eul Kgun Yum," \\ Duk Keun An, ${ }^{\ddagger}$ Joong-Kwon Choi, and Sung Soo Kim \\ Medicinal Science Division, Korea Research Institute of Chemical Technologn, Daejeon 305-600, Korea \\ "E-mail: jhathiakrict.re.kr \\ "Department of Chemistry, Chungnam National Lniversitw, Iustmg 305-764, Korea \\ 'Department of Chemistry, Kangwon National Lninersity, ChunChon 200-701, Korea \\ Received November 5, 2004
}

Key Words : 2-Py razoline-5-carboxylic acid derivatives. [2+3] Cycloaddition. Trimethylsilyldiazomethane

1,3-Dipolar cycloaddition reaction of $\alpha \beta$-unsaturated esters, amides or nitriles with diazomethane or trimethylsilyldiazomethane can be one of the powerful methods for the synthesis of pyrazoline building blocks. particularly, 2 pyrazoline-5-carboxylic ester (1). amide or nitrile which can be used as chiral precursors in the preparation of several unuatural anino acids ( 2 and 4$)^{1-4}$ and as building blocks. pyrazoline derivative (3), for the asymmetric synthesis.

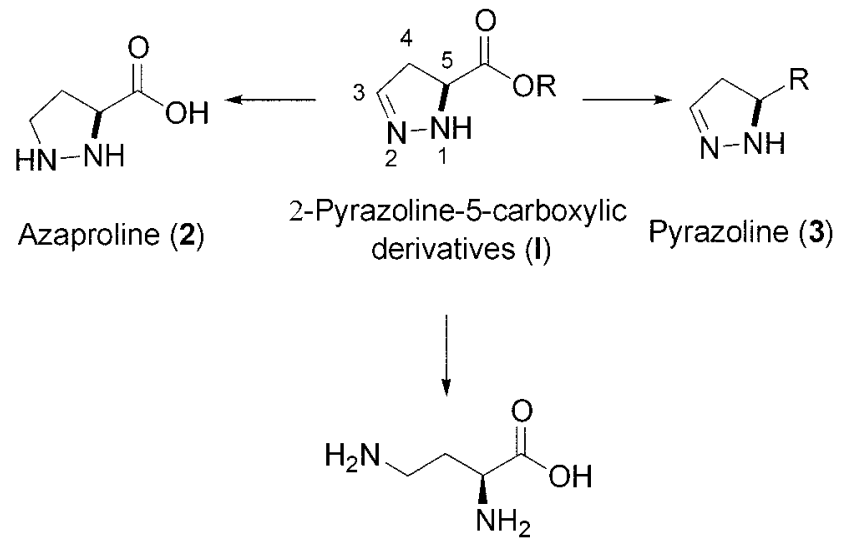

Amino Acid (4)

Scheme 1
But, prior studies $3-5$ on the cycloadditions of $\alpha . \beta$ unsaturated ester with diazoalkanes or trimethylsilyldiazomethane had indicated that the pyrazoline (5) initially formed in such cycloadditions readily isomerized (1.3proton migration) to the corresponding conjugated 2pyrazoline-3-carboxylic ester (6), not to the desired 2pyrazoline-5-carbosylic ester (1) (Scheme 2).

Also, reaction of $\alpha . \beta$-unsaturated nitrile with trimethylsily ldiazomethane gave the same result (Scheme 3$)^{6}$ and so far no single example has been reported for the cycloaddition of diazomethane or trimehtylsilyldiazomethane with $\alpha . \beta$-unsaturated amide for the synthesis of 2-pyrazoline-5-carboxamide.

These reports prompted us to attempt synthesis of 2 pyrazoline-5-carboxylic ester, amide and nitrile through controlling the reaction before 1.3-proton migration. Therefore. under several conditions, we investigated cycloaddition reaction of $\alpha . \beta$-unsaturated ester. amide and nitrile with trimethylsily ldiazomethane.

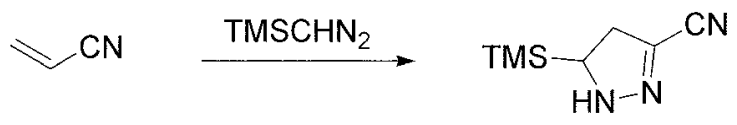

Scheme 3<smiles>[R]C=CC(=O)O[R]OC(C)C1CCCC1C(=O)O[R]</smiles>

(5)

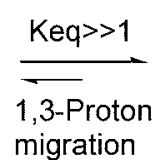

migration<smiles>[R]OC(=O)C1=NNCC1[R]</smiles>

(6)

2-Pyrazoline-3carboxylic ester

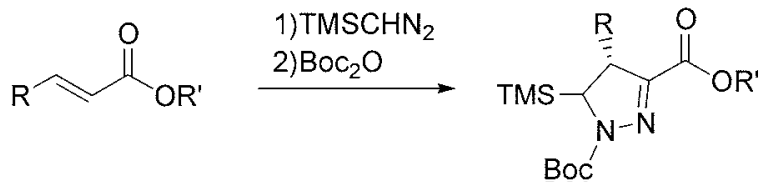<smiles>[R]C1C=NNC1C(=O)O[R]C(C)C</smiles>

(1)

2-Pyrazoline-5carboxylic ester (desired compound)

Scheme 2 
Table 1. Reaction of benzyl acrylate with trimethylsilyldiazomethane

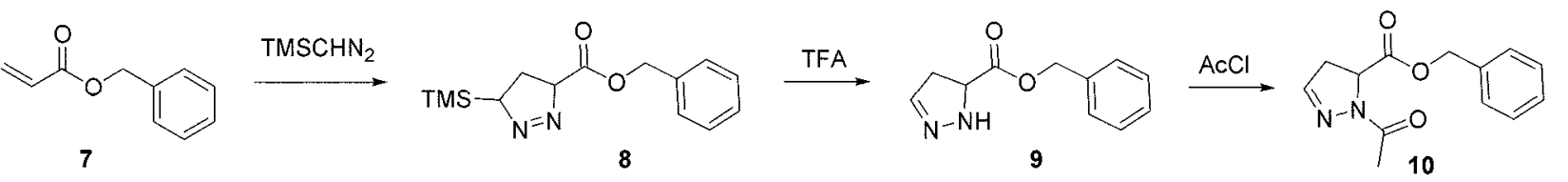

\begin{tabular}{ccccccc}
\hline Entry & Benzyl acrylate (eq.) & TMSCHN $_{2}$ (eq.) & Temp. & Rvin time & TFA (eq.) & Overall Yield (\%) \\
\hline 1 & 1 & 3 & $0^{\circ} \mathrm{C}$ & $3 \mathrm{~h}$ & 3 & 88 \\
2 & 1 & 3 & Toom temp. & $3 \mathrm{~h}$ & 3 & 35 \\
3 & 1 & 3 & $80^{\circ} \mathrm{C}$ & $3 \mathrm{~h}$ & 3 & 29 \\
4 & 1 & 2 & Toom temp. & $3 \mathrm{~h}$ & 3 & 35 \\
5 & 1 & 1.5 & Toom temp. & $3 \mathrm{~h}$ & 3 & 35 \\
6 & 1 & 1 & Toom temp. & $3 \mathrm{~h}$ & 3 & 78 \\
\hline
\end{tabular}

"Tsolated yield

As a model test. we tried the reaction of benzyl acrylate and trimethylsilyldiazomethane. and a funal compound was isolated after $N$-protection with acetyl group. The results were summarized in Table 1.

As we hoped, the reactions perfonned at $0{ }^{\circ} \mathrm{C}$ or room temperature provided the desired benzyl 2-pyrazoline-5carboxylate $(\mathbf{1 0})$ in $88 \%$ and $85 \%$ yields, respectively. (entries 1 and 2) It is noteworthy that benzyl 2-pyrazoline-5carboxylate (10) was directly synthesized from benzyl acrylate and trimethylsilyldiazomethane without 1,3migration. Whereas. the yield was decreased to $29 \%$ when the reaction was run at $80^{\circ} \mathrm{C}$. This result suggested that initially formed 1 -pyrazoline was converted to 2-pyrazoline3-carboxy lic ester and by-products at high temperature. Use

Table 2. Reaction of $\alpha, \beta$-unsaturated carbosylic derivative with $\mathrm{TMSCHN}_{2}$

Entry Substrate Time

Tsolated vield of $1.5 \mathrm{eq}$ or $1.2 \mathrm{eq}$ of trimethylsilyldiazomethane also gave satisfactory results. Based on these preliminary results, the application of this procedure to $\alpha \beta$-unsaturated esters, amide or nitrile was investigated.

As shown in Table 2. benzyl. methyl and ethyl acrylate afforded the corresponding 2-pyrazoline-5-carboxy lic esters in moderate to good yields (entries 7-9, 80-91\%). Acrylanide cyclized to give 2-pyrazoline-5-amide in $60 \%$ yield. (entry 10) Also, acrylonitrile was smoothly converted to the corresponding 2-pyrazoline-5-nitrile. (entry 11)

Next stage was the adoption of clirality at 5-position of 2pyrazoline-5-ester. There have been reports by two groups that chiral alkene (alkenoyl oxazolidinone ${ }^{7}$ or ankenoyl sultam $^{4}$ ) was treated with trimethylsilyldiazomethane to yield chiral 5-substituted pyrazoline, but they used an expensive camphorsultam ${ }^{4}$ or commercially unavailable ligand (DBFOX/Pl). ${ }^{7}$ Therefore. we investigated the reaction of chiral $\alpha . \beta$-unsaturated ester with trimethylsilyl diazomethane.

As shown in Table 3. when $(I S .2 R, 5 S)-(+)$-menthol was used as a chiral auxiliary. although none of diastereoselectivity was obtained $(1: 1)$. both of diastereomers were easily separated by colunun chromatography. To determine the absolute configuation. NOE experiments carried out with diastereomeric $15 \mathrm{a}$ and $\mathbf{1 5 b}$.

Table 3. Reaction of $\alpha \beta$-unsaturated carbosylic derivative with $\mathrm{TMSCHN}_{2}$

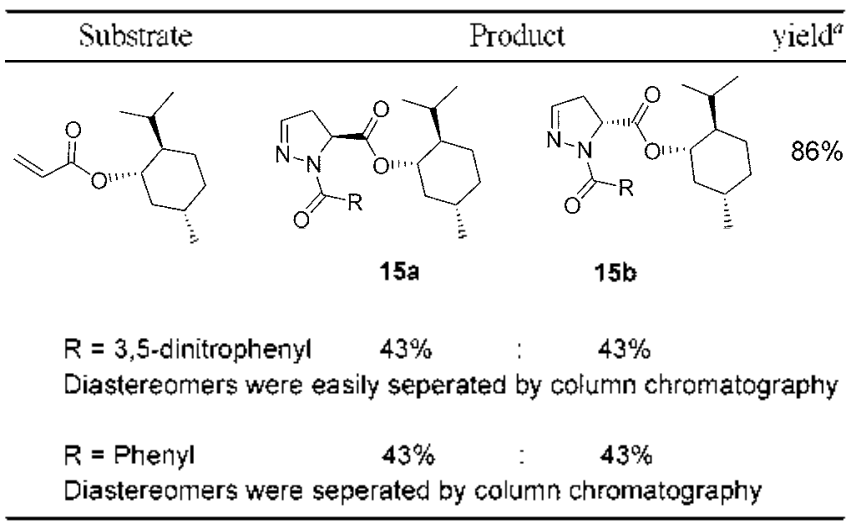



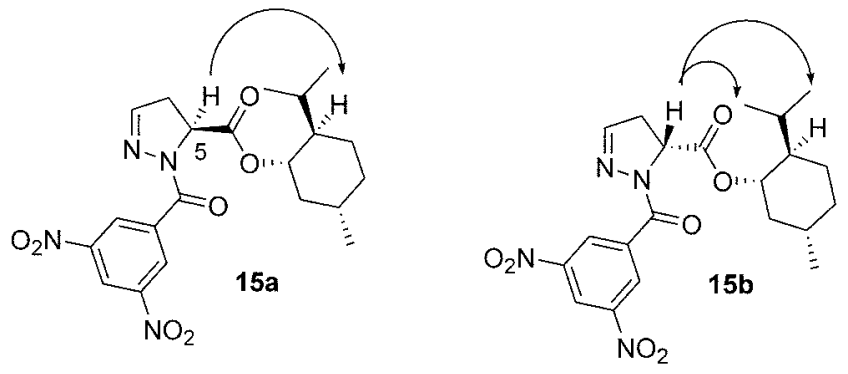

In the case of compound $15 \mathbf{a}$, the proton at $\mathrm{C5}$ of pyrazoline ring and methine proton of menthol showed a positive NOE effect $(0.96 \%)$. Also. in $15 \mathrm{~b}$, the proton at $\mathrm{C} 5$ of pyrazolin ring and methyl proton of menthol indicated a NOE effect. So. pyrazoline $\mathbf{1 5} \mathbf{a}$ and $\mathbf{1 5} \mathbf{b}$ possess $S$ and $R$ configuration. respectively

Meanwhile, using (-)-8-phenylmenthol. under the sane reaction conditions. diasteroselectivtiy was $6: 4$. (data not show11)

In conclusion. we have directly synthesized 2-pyrazoline5-carboxylic ester. anide and nitrile using trimethylsilyldiazomethane from the corresponding $\alpha, \beta$-unsaturated ester. anide and nitrile.

\section{Experimental Procedure}

Typical procedure for the preparation of 2-pyrazoline5-carboxylic derivatives. To a solution of benzyl acrylate (7) $(100 \mathrm{mig} .1 .0 \mathrm{mmol})$ in toluene $(5 \mathrm{~mL})$ was added trimethy lsilyldiazomethane $(1.5 \mathrm{~mL}, 1.5 \mathrm{mmol})$ at roon temperature. After being stirred for $3 \mathrm{~h}$, the reaction mixture was evaporated. The resulting oil was dissolved in $\mathrm{CH}_{2} \mathrm{Cl}_{2}$ and trifluoroacetic acid $(0.11 \mathrm{~mL}, 3.0 \mathrm{mmol})$ was added at 0 ${ }^{\circ} \mathrm{C}$. After being stirred for $1 \mathrm{~h}$, the reaction mixture was evaporated. The resulting oil was dissolved in $\mathrm{CH}_{2} \mathrm{Cl}=$ and acetyl chloride $(0.11 \mathrm{~mL}, 1.50 \mathrm{~mm}$ ol $)$ and triethylamine $(0.28 \mathrm{miL} .2 .0 \mathrm{nmmol})$ were added at $0^{\circ} \mathrm{C}$. After being stirred for $1 \mathrm{~h}$. the mixture was quenched with water and extracted with $\mathrm{CH}_{2} \mathrm{Cl}_{2}$. The organic layer was dried with $\mathrm{MgSO}_{4}$ and concentrated in vacuo. The residue was purified by column chromatography to afford benzyl 1-acetyl-4,5-dihydro- $\mathbf{H}$ pyrazole-5-carboxylate (10) (120 mg. 80\%): ${ }^{\text {H }}$ NMR (200 $\left.\mathrm{MHz} \mathrm{CDCl}_{\mathfrak{j}}\right) \delta 7.35(\mathrm{~s}, 5 \mathrm{H}) 6.85(\mathrm{~s}, 1 \mathrm{H}) 5.19(\mathrm{t}, J=13.5$ $\mathrm{Hz}, 2 \mathrm{H}$ ) 4.85 (dd, $J=12.3 .6 .0 \mathrm{~Hz} .1 \mathrm{H}$ ) 3.23 (d, $J=18.6$. $12.6 \mathrm{~Hz}, 1 \mathrm{H}) 2.91$ (dd. $J=18.6,5.7 \mathrm{~Hz}, 1 \mathrm{H}) 2.34$ (s. $3 \mathrm{H})$ : mass spectrum me (relative intensity) $246\left(\mathrm{M}^{-} .5\right)$ lll (29) $69(100)$.

2-Acetyl-3,4-dihydro-2H-pyrazole-3-carboxylic acid methyl ester (11): ${ }^{\mathrm{j}} \mathrm{H}$ NMR (200 MHz, CDCl 3 ) $\delta 6.86$ (s. lH) 4.79 (dd. $J=12.4$. $6.1 \mathrm{~Hz} .1 \mathrm{H}$ ) 3.75 (s. $3 \mathrm{H}$ ) 3.24 (ddd. $J$ $=18.7 .12 .4 .1 .6 \mathrm{~Hz}, 1 \mathrm{H}$ ) 2.94 (ddd. $J=18.7 .6 .3 .1 .8 \mathrm{~Hz}$. 1H) 2.33 (s. 3H): mass spectrum $m e$ (relative intensity) 170 $\left(\mathrm{M}^{+}, 12\right) 111(17) 69(100)+3(38)$.
2-Acetyl-3,4-dihydro-2H-pyrazole-3-carboxylic acid ethyl ester (12); ${ }^{1} \mathrm{H}$ NMR $\left(200 \mathrm{MHz}, \mathrm{CDCl}_{3}\right) \delta 6.87$ (s. lH) $4.79(\mathrm{dd}, J=12.3,6.0 \mathrm{~Hz} . \mathrm{lH}) 4.72(\mathrm{q}, J=14.1 .6 .9 \mathrm{~Hz} .2 \mathrm{H})$ 3.25 (dd, $J=18.6,12.6 \mathrm{~Hz}, 1 \mathrm{H}) 2.95$ (dd. $J=18.6 .5 .7 \mathrm{~Hz}$, IH) 2.34 (s. 3H) 1.29 (t. $J=7.2 \mathrm{~Hz}, 3 \mathrm{H})$; mass spectrum me (relative intensity) $184\left(\mathrm{M}^{-}, 6\right) 169$ (2) 111 (16) 69 (100) 43 (29).

2-Acetyl-3,4-dihydro-2H-pyrazole-3-carboxylic acid amide (13); ${ }^{1} \mathrm{H}$ NMR (200 MHz. DMSO-dij $\delta 7.49$ (brs, 1H) $7.07-7.06$ (n. $2 \mathrm{H}$ ) 4.54 (dd. $J=12.3 .7 .6 \mathrm{~Hz}, 1 \mathrm{H}$ ) 3.19 (ddd, $J=18.8,12.1 .1 .7 \mathrm{~Hz} . \mathrm{HH}) 2.81$ (ddd, $J=18.8,5.9,2.1$ Hz. $1 \mathrm{H}) 2.16(\mathrm{~s}, 3 \mathrm{H})$.

2-Acetyl-3,4-clihydro-2H-pyrazole-3-carbonitıile (14): ${ }^{1} \mathrm{H}$ NMR $\left(200 \mathrm{MHz} . \mathrm{CDCl}_{3}\right) \delta 6.96(\mathrm{~s}, 3 \mathrm{H}) 5.00(\mathrm{dd}, J=$ 10.5. $7.7 \mathrm{~Hz}, 1 \mathrm{H}) 3.34-3.28(\mathrm{~m}, 2 \mathrm{H}) 2.33(\mathrm{~s}, 3 \mathrm{H})$ : mass spectrum me (relative intensity) $137\left(\mathrm{M}^{+} .10\right) 84(41) 43$ (100).

2-(3,5-Dinitrobenzoyl)-3,4-dihydro-2H-pyrazole-3-calboxylic acid 2-isopropyl-5 methylcyclohexyl ester (15a): ${ }^{1} \mathrm{H}$ NMR $\left(200 \mathrm{MHz}, \mathrm{CDCl}_{3}\right) \delta 9.16($ d. $J=2.0 \mathrm{~Hz} .1 \mathrm{H}) 9.12$ (d. $J=2.0 \mathrm{~Hz}, 2 \mathrm{H}) 7.08(\mathrm{~s}, \mathrm{lH}) 5.05$ (dd. $J=12.0,5.6 \mathrm{~Hz}$, IH) 4.79 (dt, $J=10.8,4.4$ Hz. lH) 3.34 (ddd. $J=19.3 .12 .4$, $1.6 \mathrm{~Hz} . \mathrm{lH}$ ) 3.01 (ddd, $J=18.9,5.6,1.6 \mathrm{~Hz} .1 \mathrm{H}$ ) $2.06-1.92$ (m, 2H) 1.75-1.67 (m, 3H) 1.55-1.43 (m, 3H) 1.39-1.05 (m, IH) $0.95-0.80(\mathrm{~m}, 10)$; mass spectrum me (relative intensity) $237\left(\mathrm{M}^{+} .15\right) 195$ (18) $83(69) 43(100)$.

(15b): ${ }^{1} \mathrm{H}$ NMR (200 MHz. $\left.\mathrm{CDCl}_{3}\right) \delta 7.09$ (s. lH) 5.03 (dd. $J=12.0 .6 .0 \mathrm{~Hz} . \mathrm{lH}) 4.77(\mathrm{dt} J=10.8 .4 .4 \mathrm{~Hz}, \mathrm{lH})$ 3.41 (ddd $J=18.9 .5 .6 .1 .6 \mathrm{~Hz} .1 \mathrm{H}) 3.03(\mathrm{ddd}, J=18.9 .5 .6$, $1.6 \mathrm{~Hz} .1 \mathrm{H}) 2.12-2.05(\mathrm{~m}, \mathrm{lH}) 1.82(\mathrm{dt}, J=6.8,2.8 \mathrm{~Hz}, \mathrm{lH})$ $1.74-1.66$ (m. $3 \mathrm{H}$ ) $1.55-1.36$ (m. $2 \mathrm{H}$ ) 1.21 (d. $J=19.7 \mathrm{~Hz}$, IH) $1.07(\mathrm{~d}, J=10.8 \mathrm{~Hz}$. IH) 0.98-0.76 (m, 9H): mass spectrum me (relative intensity) $237\left(\mathrm{M}^{-} .11\right.$ ) 195 (19) 138 (34) $83(100)$.

Acknowledgment. This research was supported by the Center for Biological Modulators of the 21 st Century Frontier R\&D Program. the Ministry of Science and Teclunology. Korea.

\section{References}

1. Fuchi. N.: Doi. T.: Harada. T.: Urbant. T.: Cao. B.: Kahn. M.: Takahashi. T. Tetrohedton Lett. 2001. 42.1305.

2. Kiml. H. O.: Lum. C.: Lee. M. S. Tetrahedron Lett. 1997. 38. 4935.

3. Whitlock, G. A.; Carreira, E. M. Helv Chim Acta 2000. 83. 2007

4. Mish. M. R. Guerra. F. M.; Carreira, E. M. J. Am. Chem. Soc. 1997. 119.8379.

5. Barluenga. J.: Fernandes-Mari. F.: Viado. A. L.: Aguilar. E.: Olano. B.: Garcia-Granda. S.: Moya-Rubiera. C. Chem. Em: J. 1999. 5,883 .

6. Seyferth, D.; Dow: A. W.: Menzel, H; Flood. T. C. J. Am. Chem. Soc. 1968, 90, 1080.

7. Kanemasa. S.: Kan1ai. T. J. Ant Chent Soc. 2000. 122. 10710. 